\title{
Design of a Biomechanical Model and a Set of Neural Networks for Monitoring of Weightlifting
}

\author{
Francisco Javier Rosas Ibarra, María Trinidad Serna Encinas, \\ César Enrique Rose Gómez, and Oscar Adrián Pinto García \\ Instituto Tecnológico de Hermosillo, División de Estudios de Posgrado e Investigación, \\ Maestría en Sistemas Industriales, \\ Av. Tecnológico S/N, Hermosillo, Sonora, México. \\ \{frosas, tserna, crose\}@ith.mx, elpintojregmail.com
}

\begin{abstract}
Nowadays, young athletes have injuries that leave them out the competitions, this injuries may occur due to the lack of the basic principles of weight training, muscle imbalance, fatigue, overhead, among others. The proposed weightlifting monitoring system is intended to coaches in order to provide them better control of the athletes during the training session, through continuous monitoring of exercise's measurements. This allows to detect certain risks of injury that may result if an exercise is poorly executed. To handle the uncertainty generated by the comparison of data obtained from athlete with the data of the biomechanical model for weightlifting, a set of neural networks to classify the athletes' movements during training is used. This paper describes the biomechanical model and the proposed neural network design for such monitoring system.
\end{abstract}

Keywords: Monitoring system, Weightlifting, Neural networks, Biomechanical model.

\section{Introduction}

In sports training, who is responsible for athletes to perform the right moves is the coach, but if he is in charge of several athletes at the same time, he may not notice that someone is performing wrong an exercise, or may fail to distinguish when an exercise is being poorly executed.

The monitoring system, that is sought to implement, will be used with elite/professionals athletes of the Sonora Sports Commission (CODESON), the system take measurements of the exercises carried out in weightlifting, using Kinect ${ }^{\circledR}$ sensors in a controlled environment. These measurements will be compared with a biomechanical model for weightlifting defined with specific movements for this exercise, and will be classified by a set of neural networks to determine if the positions of the executed exercise correspond to the positions of the base pattern previously defined and which is the precision level of the movements. 
This paper focuses on the design of the set of neural networks to the monitoring system for the weightlifting training. Section 2 briefly presents the biomechanical model and the set of parameters defined in each phase of the exercise; Section 3 describes the proposed design of the neural network; specifically, it shows in detail the neural network for Phase 1. Section 4 discusses about the expected results; and finally, Section 5 presents the conclusion of this research.

\section{Biomechanics Model}

Sports biomechanics arises as a result of the development of the biomechanics of physical exercise, and was created by P. F. Lesgaft in the second half of the nineteenth century, it is responsible for evaluating a sport activity in order to design training techniques, improve performance and avoid injury [1].

A biomechanical model is a structure that represents the relationship between the objectives of the skills and the factors that produce those skills [2].

In Figure 1 the biomechanical model for weightlifting is shown, which is divided into six phases: Phase 1 (Initial position), which takes into consideration the angles of the ankles, knees, trunk and neck; Phase 2 (First pull), in addition to taking the angles also analyzes the horizontal scroll bar, vertical speed, maximum height of the bar and time; in Phase 3 (Adjustment) only the vertical velocity and time is analyzed; Phase 4 (Second pull) analyzed the same variables that Phase 2; Stage 5 (Slip) takes into account the time and bar height that it can reach in different types of lifts; for instance, slip unsupported (snatch) or slip supported (clean and jerk); Finally, in Phase 6 (Recovery), recovery height and position of the torso, arms and legs is analyzed [2-3].

\subsection{Biomechanical Model with Parameters}

In Figure 2 the biomechanical model for weightlifting with the parameters defined in each phase is observed. For Phase 1 the ankle angles must be $50^{\circ}$, the knee angles should be $72^{\circ}$, the trunk angle should be $45^{\circ}$ and the neck angle should be $160^{\circ}$.

In Phase 2 ankle angles should be $25^{\circ}$, the knee angles should be $149^{\circ}$, the trunk angle should be $79^{\circ}$ and the neck angle should be $160^{\circ}$; the horizontal scroll bar should be between -0.03 and $-0.13 \mathrm{~m}$; vertical velocity should be 1 to $1.6 \mathrm{~m} / \mathrm{s}$; the maximum height of the bar should be $28 \%$ to $32 \%$ of the height of the athlete; and time should be between 0.35 and 0.64 seconds.

For Phase 3, the vertical velocity should be between 0.88 and $1.52 \mathrm{~m} / \mathrm{s}$; and time should be between 0.08 and 0.19 seconds [2-5].

In phase 4 the angles of the ankle must be $32^{\circ}$, the knee angles should be $163^{\circ}$, the trunk angle must be $170^{\circ}$ and $175^{\circ}$ the angle of the neck; the horizontal scroll bar must be between 0 and $-0.11 \mathrm{~m}$; the vertical velocity should be 1.6 to 2.5 meter/second; the maximum height of the bar should be $60 \%$ to $66 \%$ of the height of the athlete, and time should be between 0.11 and 0.21 seconds.

For Phase 5, the time should be between 0.25 and 0.63 seconds; the height of the bar in the slip without support should be between $62 \%$ and $75 \%$ of the athlete's 


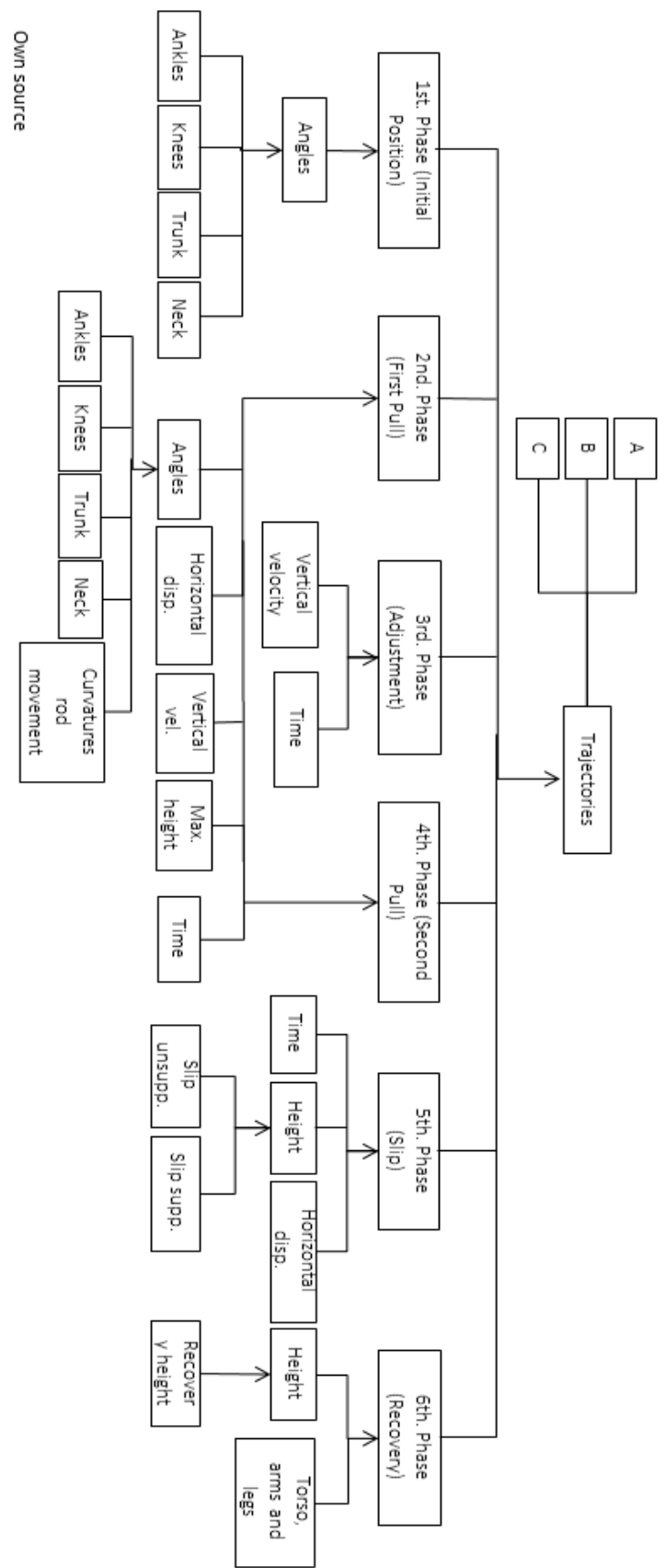

Fig. 1. Biomechanical model for weightlifting composed of 6 phases.

height, and bar height to support the slip should be between $75 \%$ and $95 \%$ of the athlete's height, and the horizontal scroll bar should be between -0.02 and $-0.10 \mathrm{~m}$. 
Francisco Javier Rosas Ibarra, María Trinidad Serna Encinas, César Enrique Rose Gómez, et al.

For Phase 6 height recovery should be $106 \%$ and the position of the torso, arms and legs should be $180^{\circ}$.

\subsection{Definition of Ranges}

The definition of ranges is carried out taking into account the coaches, and even though they are aware that there is no perfect movement, it is desirable that athletes come close to it frequently. This was the reason that forced define ranges for each of the parameters that are in the biomechanical model previously described, and that are used by the set of neural networks to classify movements performed during training. The ranges used are those that occur especially in the angles and the degrees of freedom to allow in motion, so that it remains acceptable performance.

For example, in the knee angles of Phase 1 , the parameter specifies $72^{\circ}$. The stated range for this parameter is $\pm 3^{\circ}$, i.e., the optimal motion varies between $69^{\circ}$ and $75^{\circ}$, the acceptable movement is between $66^{\circ}$ and $69^{\circ}$ or between $75^{\circ}$ and $78^{\circ}$, and the movement that is not acceptable is below $66^{\circ}$ and above $78^{\circ}$.

\section{Artificial Neural Network}

An artificial neural network (ANN) is a set of simple units called neurons connected together by means of connections, and each element works only with local information and asynchronously. The knowledge is stored in the connections or synaptic weights, these weights will be adjusted according to the patterns that are available through a learning rule, this part of the process, in which the ANN learns the patterns, it is known as training. The knowledge acquired in this phase is reflected in certain values of the synaptic weights, with which the network must be able to generalize; that is, to give the correct output to a given input previously unseen [6-9].

Figure 3 shows the hierarchical structure of an ANN based system which begins with the neuron as is shown, then the layer is, which is a set of neurons in the same level, then the network itself is, a set of layers, and finally, the neuronal system containing inputs, a network, an algorithmic part and an output layer.

\subsection{Design of the Neural Network Proposed to Monitoring System}

The set of neural networks are designed based on the phases of the biomechanical model for weightlifting, that is, six neural networks, one for each phase, to allow classification of the movement, considering the ranges defined for each parameter as outputs of the set of networks.

\subsection{Creation of the Neural Network}

Figure 4 shows the neural network of Phase 1 that includes six neurons in the input layer, six neurons in the hidden layer, and six neurons in the output layer. For the 


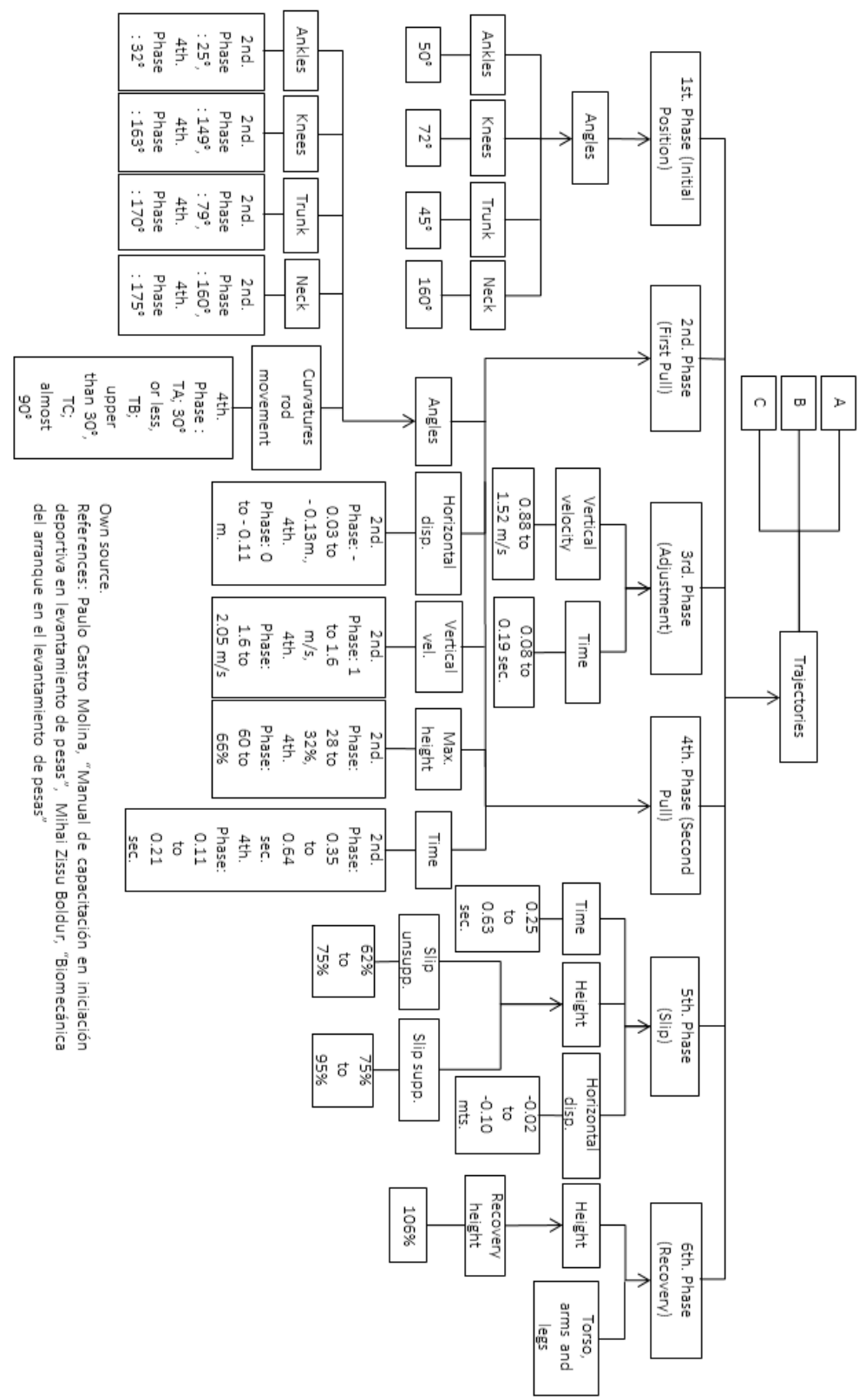

Fig. 2. Biomechanical model for weightlifting with parameters.

inputs we consider the angles of right and left knee ( 2 inputs), the right and left ankle ( 2 inputs), trunk ( 1 input) and neck ( 1 input), which make up the six input neurons. 


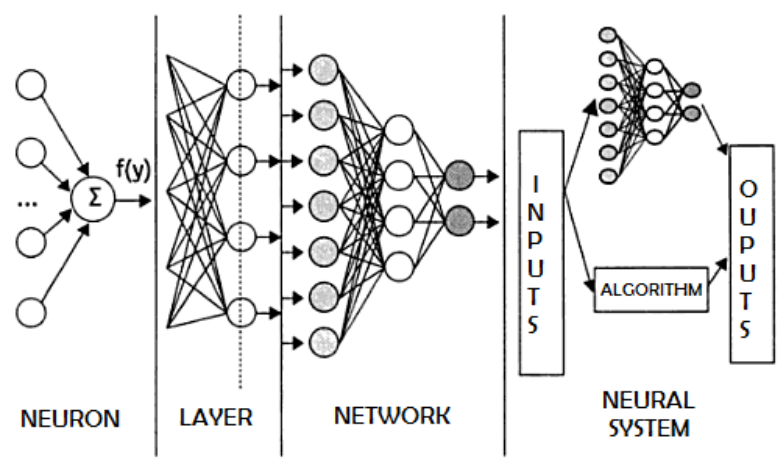

Fig. 3. Hierarchical structure of an ANN based system.

For each input has an output; i.e., an output for the right knee, one for the left knee, one for the right ankle, one for the left ankle, one for the trunk, and one for the neck, giving the six expected outputs.

Figure 5 shows the neural networks of Phase 2 and Phase 4 that includes eleven neurons in the input layer, eleven neurons in the hidden layer, and eleven neurons in the output layer. Besides consider the six angles mentioned before as inputs, it also has the bend of the bar (1 input), the horizontal scroll (1 input), the vertical speed (1 input), the maximum bar height (1 input) and time (1 input), which make up the eleven input neurons. Also it has an output for each input in Phase 2, making eleven expected outputs.

The neural network for Phase 3 only considers two inputs, which are vertical velocity ( 1 input) and time ( 1 input). It has two outputs, one for each input, giving the two expected outputs, has shown in figure 6 .

Figure 7 shows he neural network for Phase 5 that considers three inputs, time (1 input), maximum bar height (1 input) and horizontal scroll (1 input). For each input there is an output, so there are three outputs on Phase 5.

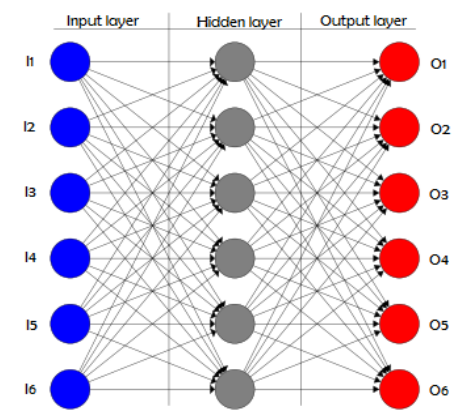

Fig. 4. Design of the neural network for the phase 1 (Initial position). 


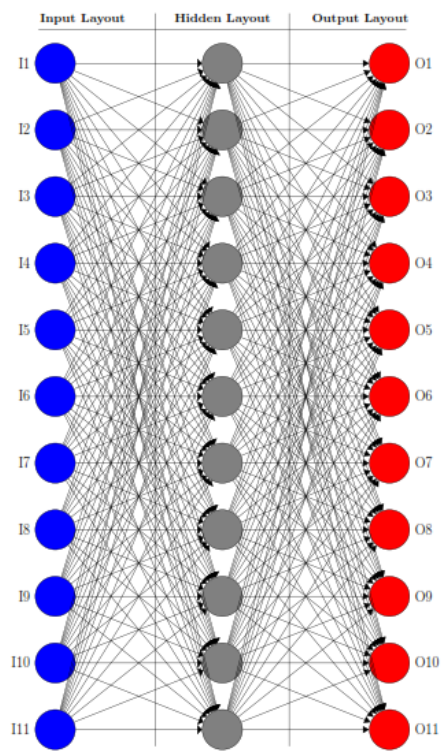

Fig. 5. Design of the neural network for phases 2 and 4 (First and second pull).

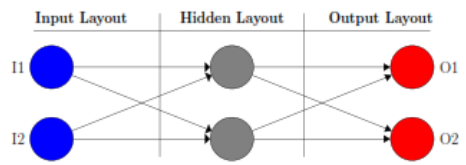

Fig. 6. Design of the neural network for the phase 3 (Adjustment).

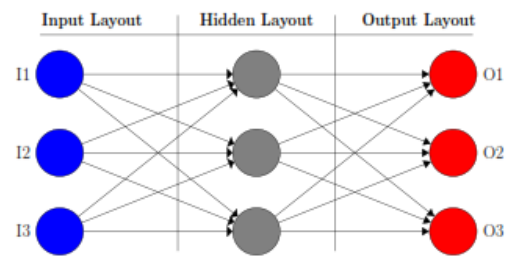

Fig. 7. Design of the neural network for the phase 5 (Slip).

Figure 8 shows the neural network of Phase 6 that has six inputs, the left and right knee angle ( 2 inputs), the trunk angle ( 1 input), the neck angle ( 1 input), the horizontal scroll ( 1 input) and the maximum bar height (1 input). There are six outputs in this neural network, one for each input.

The inputs of each phase correspond to the parameters set in each of the phases of the previously described model. 


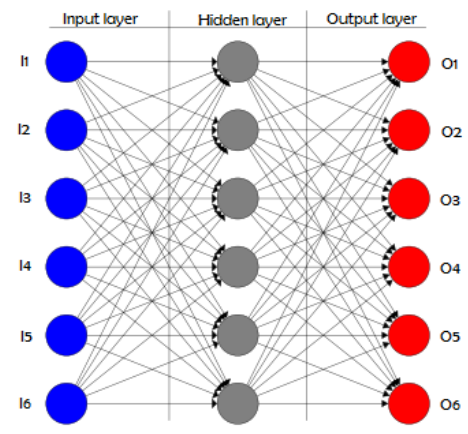

Fig. 8. Design of the neural network for the phase 6 (Recovery).

The activation function used in the neural network is set bipolar sigmoidal with alpha value equal to 0.5 . Next is shown the part of the code that creates the neural network by assigning the number of inputs, outputs, the activation function and for neurons in the hidden layer.

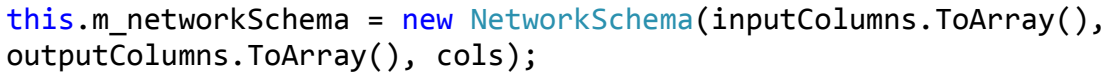

\subsection{Training the Neural Network}

The really important feature of neural networks is the ability to learn and adapt to the environment. The training process that makes that a neuron learn is vital part of the system and determines the degree of intelligence that can acquire the neural network [7].

For training the neural network of Phase 1 was required to record the movements of several athletes, from a very refined technique athlete, which performed the best moves according to the coach, and with another athlete with a less refined technique and with some mistakes.

In Table 1 are shown some of the input and output data used for training the neural network of Phase 1, which can be seen as inputs that have LeftAnkle_Angle, RightAnkle_Angle, LeftKnee_Angle, RightKnee_Angle, Neck_Angle and CenterHip_Angle; and outputs are O1, O2, O3, O4, O5 and O6. The outputs are taken at a range of 1 to 5, for example, for knees the 1 is angle less than $66^{\circ}$ (not acceptable), a 2 is between $66^{\circ}$ and $69^{\circ}$ (acceptable), a 3 when it is between $69^{\circ}$ and $75^{\circ}$ (optimal), a 4 is between $75^{\circ}$ and $78^{\circ}$ (acceptable), and finally, 5 is an angle greater than $78^{\circ}$ (not acceptable) angle. 
In Table 2 are shown some of the input and output data used for training the neural network of Phase 1, which can be seen as inputs that have LeftAnkle_Angle, RightAnkle_Angle, LeftKnee_Angle, RightKnee_Angle, Neck_Angle, CenterHip_Āngle, Curvature_Movement, Hor_Scroll, Vertical_Vel, Time and Maximum_Height.

Table 1. Input data and output data for the training of the neural network of the Phase 1 (Initial Position).

\begin{tabular}{cccccccccccc}
\hline $\begin{array}{c}\text { LAnkle } \\
\text { Angle }\end{array}$ & $\begin{array}{c}\text { RAnkle } \\
\text { Angle }\end{array}$ & $\begin{array}{c}\text { LKnee } \\
\text { Angle }\end{array}$ & $\begin{array}{c}\text { RKnee } \\
\text { Angle }\end{array}$ & $\begin{array}{c}\text { Neck } \\
\text { Angle }\end{array}$ & $\begin{array}{c}\text { CHip } \\
\text { Angle }\end{array}$ & O1 & O2 & O3 & O4 & O5 & \multirow{2}{*}{ O6 } \\
\hline 13.429 & 4.301 & 85.808 & 155.39 & 160.26 & 139.77 & 4 & 4 & 4 & 4 & 4 & 4 \\
6.081 & 2.205 & 93.699 & 56.080 & 172.50 & 132.19 & 4 & 4 & 4 & 4 & 4 & 4 \\
5.594 & 2.041 & 94.077 & 55.371 & 171.84 & 132.19 & 4 & 4 & 4 & 4 & 4 & 4 \\
6.491 & 2.284 & 93.273 & 57.063 & 172.87 & 132.29 & 4 & 4 & 4 & 4 & 4 & 4 \\
14.503 & 26.545 & 60.689 & 41.487 & 159.84 & 59.599 & 3 & 3 & 3 & 3 & 3 & 3 \\
39.816 & 14.878 & 61.813 & 38.131 & 158.16 & 57.71 & 3 & 3 & 3 & 3 & 3 & 3 \\
17.213 & 21.445 & 57.364 & 40.883 & 157.87 & 60.976 & 3 & 3 & 3 & 3 & 3 & 3 \\
24.058 & 45.633 & 65.514 & 46.659 & 158.77 & 72.077 & 3 & 3 & 3 & 3 & 3 & 3 \\
51.751 & 22.979 & 60.922 & 54.889 & 160.32 & 81.226 & 3 & 3 & 3 & 3 & 3 & 3 \\
\hline
\end{tabular}

Table 2. Input data for the training of the neural network of the Phase 2 (First Pull).

\begin{tabular}{ccccccccccc}
\hline $\begin{array}{c}\text { LAnk } \\
\text { Angle }\end{array}$ & $\begin{array}{c}\text { RAnk } \\
\text { Angle }\end{array}$ & $\begin{array}{c}\text { LKnee } \\
\text { Angle }\end{array}$ & $\begin{array}{c}\text { RKnee } \\
\text { Angle }\end{array}$ & $\begin{array}{c}\text { Neck } \\
\text { Angle }\end{array}$ & $\begin{array}{c}\text { CHip } \\
\text { Angle }\end{array}$ & $\begin{array}{c}\text { Hor } \\
\text { Scroll }\end{array}$ & $\begin{array}{c}\text { Vert } \\
\text { Vel }\end{array}$ & \multirow{2}{*}{ Time } & $\begin{array}{c}\text { Max } \\
\text { Heig }\end{array}$ & $\begin{array}{c}\text { Curv } \\
\text { Mov }\end{array}$ \\
\hline 15.932 & 17.883 & 92.617 & 95.074 & 139.24 & 152.34 & 0.35 & 0.921 & 0.664 & 0.351 & 32.145 \\
9.711 & 12.378 & 89.271 & 91.871 & 140.36 & 153.94 & 0.38 & 0.873 & 0.675 & 0.348 & 31.587 \\
13.492 & 15.301 & 85.808 & 88.332 & 145.78 & 148.21 & 0.54 & 0.869 & 0.677 & 0.337 & 31.986 \\
13.241 & 15.856 & 86.237 & 88.024 & 143.25 & 142.37 & 0.73 & 0.935 & 0.688 & 0.322 & 33.754 \\
26.856 & 28.545 & 58.189 & 60.297 & 160.84 & 90.599 & -0.01 & 1.12 & 0.38 & 0.283 & 23.541 \\
31.189 & 34.578 & 59.093 & 61.031 & 159.16 & 87.071 & 0.01 & 1.28 & 0.39 & 0.287 & 28.152 \\
41.997 & 43.743 & 63.574 & 65.753 & 158.97 & 87.976 & 0.03 & 1.35 & 0.45 & 0.292 & 27.378 \\
48.723 & 51.683 & 65.348 & 66.659 & 158.13 & 78.573 & 0.08 & 1.42 & 0.47 & 0.301 & 29.961 \\
\hline
\end{tabular}

Table 3. Output data for the training of the neural network of the Phase 2 (First Pull).

\begin{tabular}{ccccccccccc}
\hline $\mathrm{O} 1$ & $\mathrm{O} 2$ & $\mathrm{O} 3$ & $\mathrm{O} 4$ & $\mathrm{O} 5$ & $\mathrm{O} 6$ & $\mathrm{O} 7$ & $\mathrm{O} 8$ & $\mathrm{O} 9$ & $\mathrm{O} 10$ & $\mathrm{O} 11$ \\
\hline 4 & 4 & 4 & 4 & 4 & 4 & 4 & 4 & 4 & 4 & 4 \\
4 & 4 & 4 & 4 & 4 & 4 & 4 & 4 & 4 & 4 & 4 \\
4 & 4 & 4 & 4 & 4 & 4 & 4 & 4 & 4 & 4 & 4 \\
4 & 4 & 4 & 4 & 4 & 4 & 4 & 4 & 4 & 4 & 4 \\
3 & 3 & 3 & 3 & 3 & 3 & 3 & 3 & 3 & 3 & 3 \\
3 & 3 & 3 & 3 & 3 & 3 & 3 & 3 & 3 & 3 & 3 \\
3 & 3 & 3 & 3 & 3 & 3 & 3 & 3 & 3 & 3 & 3 \\
3 & 3 & 3 & 3 & 3 & 3 & 3 & 3 & 3 & 3 & 3 \\
\hline
\end{tabular}




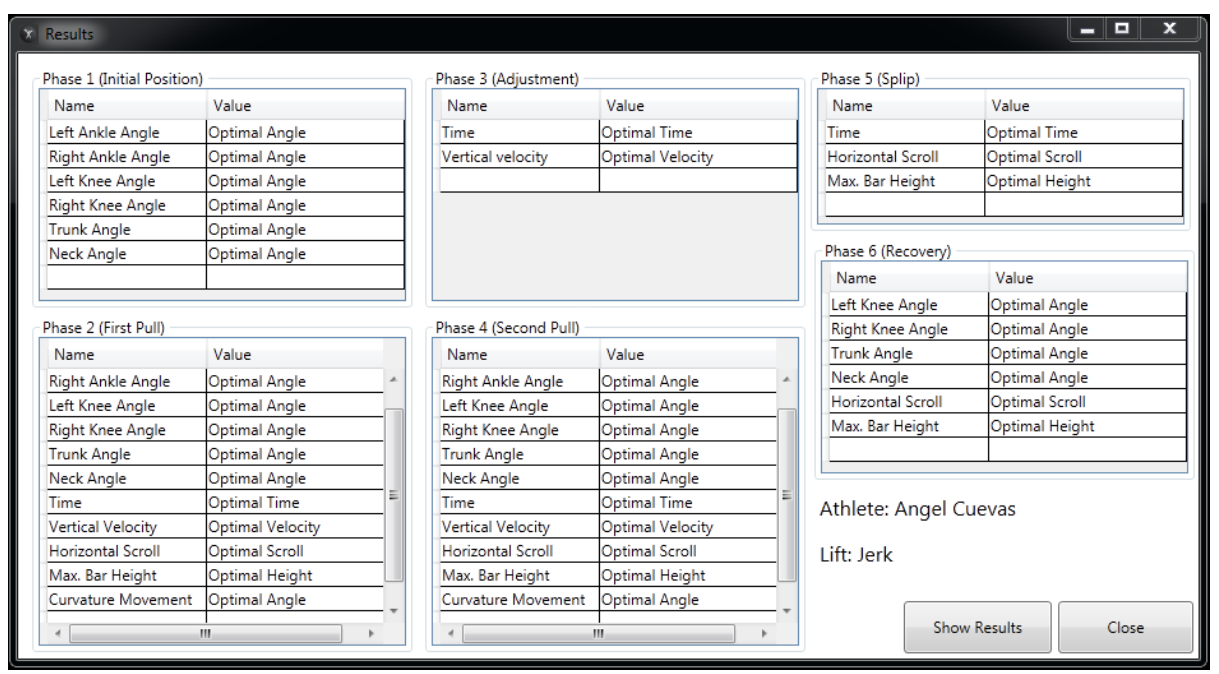

Fig. 9. Results obtained in jerk for an athlete with refined technique.

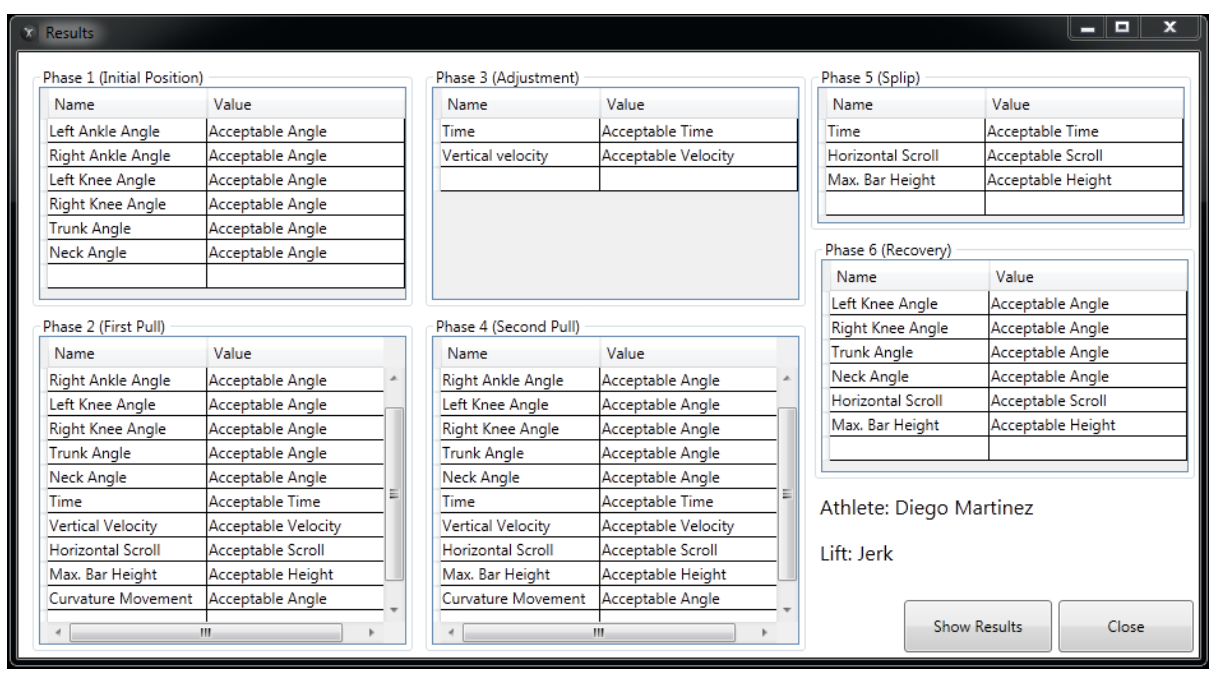

Fig. 10. Results obtained in jerk for an athlete with no refined technique.

In Table 3 are shown some of the outputs using a range of 1 to 5, for example, for horizontal scroll the 1 is scroll less than -0.18 (not acceptable), a 2 is between -.18 and -0.13 (acceptable), a 3 when it is between -.013 and 0.3 (optimal), a 4 is between 0.3 and 0.8 (acceptable), and finally, 5 is a scroll greater than 0.8 (not acceptable) scroll.

Phase 4 has the same input parameters than Phase 2, but with the values for the training of the Phase 4 . The Phase 3 has 2 inputs parameters, the Phase 5 has 3 input 
parameters and the Phase 6 has 6 input parameters, with the corresponding values of each phase.

For training the network a learning rate of 0.1 , a momentum of 0.0 , and the limit of error in 0.1 are used, the learning function is Backpropagation. Below is shown the code with which the network is trained:

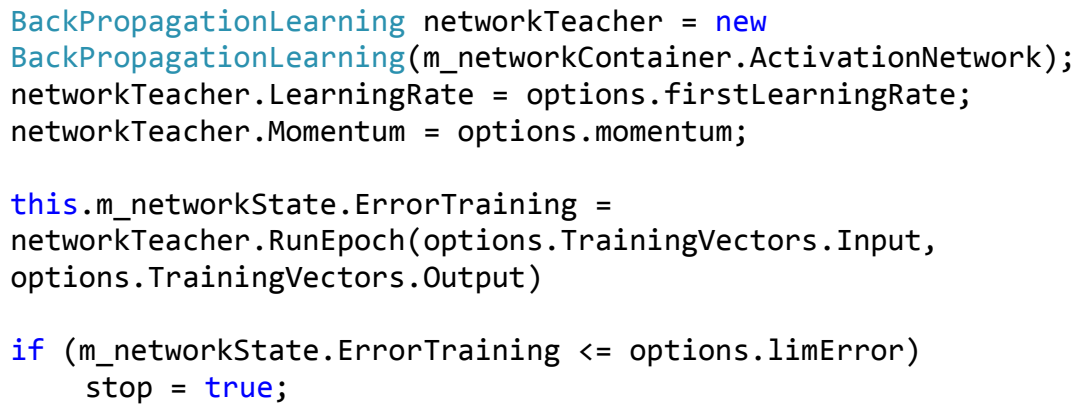

\section{Expected Results}

By integrating the outputs of the set of neural network we can know the classification of movements performed by the athlete, also we can know the movements that must be corrected and the phases in which they were executed, therefore, we expected that this help to improve his technique and avoid risk of injury.

Figure 9 shows the results obtained after the use of the set of neural networks for jerk, you can see the 6 phases with all the parameters to be analyzed (right and left knees angles, right and left ankles angles, neck and trunk angles, time, vertical velocity, horizontal scroll, maximum bar height, curvature movement, depending on each phase) and outputs for each them.

Figure 10 shows, similarly, the results after the use of the set of neural networks for jerk, where the result of the parameters of the six phases to be analyzed for an athlete with no refined technique is deployed.

\section{Conclusions}

The biomechanical model proposed for weightlifting consists of six phases (initial position, first pull, adjustment, second pull, slip and recovery). Each phase consists of a set of parameters, which specify the optimal value to achieve during the performance of movements. With the literature, it is possible to define the optimum value of approximately half of the parameters; other values were determined with the support of the coaches. Similarly, it was possible to define the range of values for each of the (optimal, acceptable and not acceptable) parameters.

Using the proposed biomechanical model for weightlifting, where the set of parameters for each phase and the ranges for each parameter are defined, we obtain a design for the set of neural networks for the classification of movements performed 
during the exercise, which will end system implementation and present the analysis of results.

Acknowledgment. We thank "Dirección General de Educación Superior Tecnológica" (DGEST) for the scholarship with number 704464, issued from August 2012 to July 2014 for the completion of a Masters in Industrial Systems.

\section{References}

1. Ford J., Ángel E.: La biomecánica deportiva en el arte marcial del full contact, Tesis de licenciatura, Universidad Central de Venezuela, Venezuela, (2010)

2. Beltrán, S. P., Boldur, M. Z.: Características cinemáticas de la fase del halón en el arranque, Levantamiento de pesas femenino. EAE Editorial Academia Española, ISBN13: 9783846567777, p. 60, España, (2011)

3. Ford, A. E.: La biomecánica deportiva en el arte marcial del full contact: Un reportaje educativo e instruccional, Ph.D. dissertation, (2013)

4. Sobarzo, P. D.: Biomecánica deportiva, Boletín informativo de la Coordinación de la Investigación Científica, el faro, no. 88 - 89, p. 8 - 10, (Agosto 2008)

5. Suárez, G. R.: Biomecánica deportiva y control del entrenamiento, Primera edición, Funámbulos Editores, Medellín, Colombia, (2009)

6. Nigrin, A., Neural networks for pattern recognition. MIT Press, (1993)

7. Isasi P., Galván I.: Redes de neuronas artificiales un enfoque práctico, Pearson Education, ISBN: 84-205-4025-0, Madrid, (2004)

8. Del Brío, B. M., Molina, A. S.: Redes neuronales y sistemas difusos, (2002)

9. Matich, D. J.: Redes Neuronales: Conceptos básicos y aplicaciones, Cátedra de Informática Aplicada a la Ingeniería de Procesos-Orientación I, (2001) 